\title{
The Photon Scanning Tunneling Microscope
}

\author{
T. L. Ferrell, J. P. Goudonnet, R. C. Reddick, \\ S. L. Sharp, and R. J. Warmack
}

\author{
Health and Safety Research Division* \\ Oak Ridge National Laboratory \\ P.O. Box 2008 \\ Oak Ridge, TN 37831-6123
}

* Sponsored by the Office of Health and Environmental Research. U.S. Department of Energy, under contract DE-AC05-84OR21400 with Martin Marietta Energy Systems. Inc.

\section{DISCLAIMER}




\section{Introduction.}

The advent of quantum mechanics in 1926 brought a unifying view of the particle-wave duality that permitted a straightforward understanding of puzzling phenomena such as tunneling. Nobel prizes awarded for applying the concept of tunneling have since included those for the theory of alpha decay, the invention of tunnel junctions, and the invention of the electron scanning tunneling microscope. Tunneling may occur when two media are separated by a third medium and a wave description is appropriate for determining the propagation of energy. If only ordinary traveling waves are required for the analysis prior to looking at tunneling, the desired results are the reflection and transmission, or refraction, coefficients at each boundary. These may be cast in terms of the angle of incidence and the relative indices of refraction. For simplicity, suppose two identical nonabsorbing media in which the traveling wave has momentum magnitude $p$ are separated by a planar nonabsorbing film in which the traveling wave's momentum has magnitude $\mathrm{p}^{\prime}$ and $\mathrm{p}^{\prime}$ is less than $\mathrm{p}$. The index of refraction is then defined to be $n=p / p^{\prime}$, a definition that can be used in quantum theory for both photons and electrons. Suppose then one imagines a wave incident on the film at angle $\theta$ with respect to the surface normal, and that the wave is simply refracted penetrating into the film with angle $\theta^{\prime}$. Also, suppose temporarily that the film is very thick relative to the wavelength. Conservation of the tangential momentum components then yields Snell's law for both electrons and photons:

$$
\frac{\sin \theta^{\prime}}{\sin \theta}=\mathrm{n}
$$

If the photons are polarized in a direction perpendicular to the plane of incidence (s-polarized light), then the same results (Fresnel's equations) are obtained for the reflection and refraction coefficients for both photons and electrons (or other particles such as alpha particles, neutrons, etc.). If the film is very thick, $\theta$ may be increased to the point that $\theta^{\prime}=90^{\circ}$ and for larger $\theta$ no energy is transmitted into the film. However, the wave has an exponentially decaying amplitude in the film. If the film thickness is reduced to the order of a wavelength, there appears an exponentially increasing amplitude in the film and energy may be propagated through. This is tunneling. That is, because a wave description is appropriate, there is a nonzero transmission through the film. The exponential dependence is dominated by the decreasing exponential in most tunneling microscopy work and the concomitant rapid decay of the signal with increasing gap is responsible for the extraordinary resolution of these microscopes both normally and tangentially. 


\section{Photon Tunneling}

Presently, there are only two types of scanning tunneling microscopes: those using electrons and those using photons. The electron scanning tunneling microscope (ESTM) is now a familiar sight in hundreds of laboratories. The exact analogue of the ESTM is the photon scanning tunneling microscope (PSTM). ${ }^{1,2}$ In the PSTM the sample is the surface at which total internal reflection of photons occurs unless an optical fiber probe tip is brought nearby. The probe tip is chemically etched to a sharp point, just as with the metal tip of an ESTM, and just as in the ESTM, the rapid exponential decay makes most of the probe irrelevant to the resolution. The "effective shape" of the probe tip is thus better than its actual shape for attaining good tangential resolution. Of course, for samples with local topographies that permit tunneling into the side of an adjacent tip, it is important to have a tip with a narrow aspect ratio, but gentler surfaces are resolved with poorer tips. Additionally, resolution is best for the ESTM for electrically conducting samples, while the best PSTM resolution is obtained on optically conducting samples. Fig. 1 shows a schematic for the PSTM and for the ESTM. It is important to note that, once the sample is coupled to the prism, the top of the sample becomes the boundary at which tunneling occurs.

Just as in the ESTM, the tip in the PSTM is typically scanned with a tubular piezoelectric scanner with feedback electronics to maintain a constant signal current. In the PSTM the photons are transmitted in the optical fiber to a photomultiplier tube to produce an electrical signal, but the electronics is identical to that of the ESTM. A bias voltage is used between the tip and the sample in the ESTM to facilitate tunneling, and comparable effects occur in the PSTM when the wavelength is changed. As noted above, the reflection and transmission coefficients for tunneling of electrons and "s-polarized" photons are identical. This is true despite the fact that the photon's wave function is a vector while the electron's wave function is a scalar. The PSTM can take advantage of the vector nature of the photon's wave function as it is a simple matter to change polarizations and for light polarized parallel to the plane of incidence ("p-polarized" light) the reflection and transmission coefficients are different from the s-polarized case.

\section{The Niche Filled by the PSTM}

In comparison with other optical microscopes the PSTM offers three-dimensional imaging with subwavelength resolution. A PSTM image of an "optically flat" quartz surtace is shown in Fig. 2. Polishing marks are clearly visible in the image and a cross-cut of the data reveals the profile quantitatively. It is possible to obtain tangential resolution of one tenth of the wavelength. 
Resolution nomal to the sample is primarily limited by the electronics and is easily one nanometer or better. Further optimization may result in a tangential resolution that is a factor of two better than the tigure just quoted, but it is also possible to simply use a smaller wavelength. Ultimately, by taking advantage of the known optical properties of selected materials, it will be possible to use soft $x$-rays and thereby obtain better than one nanometer resolution tangentially. Also, when the sample is absorbing it is convenient to change to a different wavelength.

Comparing the PSTM with ordinary electron microscopes shows that the primary advantage of the PSTM lies in examining electrically insulating samples such as biological samples. (Spectroscopic advantages are discussed in Section 5.) Electron microscopes typically require such samples to be coated with an electrically conducting layer and usually require a vacuum in the sample chamber. On the other hand, the ESTM can image thin insulating layers on a conducting substrate in a fluid environment, but there are many difficulties. The PSTM does not have these difficulties, but of course, it is not a panacea for high-resolution biological work -.- biological samples invariably pose difficult problems for all types of microscopy, including the PSTM. At this early stage, entirely new sample methodologies are needed for this important application of the PSTM. This is much as it was in the early days of transmission electron microscopy, but clearly the PSTM has a niche of its own that will permit us to see things never before seen.

Another optical microscope with subwavelength resolution is the near-field scanning optical microscope. ${ }^{3}$ This instrument uses an aperture limited optical fiber to probe the radiation from samples in the near zone. The signal is determined by the character of multipoles, the field of which induces radiative multipoles on the aperture. Resolution is as good or better than the PSTM on certain samples and, while there are some drawbacks due to the lack of a rapid exponential decay, there are important applications for this technology.

\section{PSTM Images}

We have obtained PSTM images of a variety of samples and compared them with other images of the same samples. Fig. 3a. shows a PSTM image of an optical grating formed in uncoated. transparent photoresist. The same grating was coated with silver and imaged by an ESTM (Fig. 3b) and by a SEM (ordinary scanning electron microscope: Fig. 3c). The grating spacing is $1.17 \mu \mathrm{m}$. A cross-cut of the PSTM data is shown in Fig. 4. The grating depth is seen to be about 
$140-180 \mathrm{~nm}$, depending upon the site selected on the grating. This reflects the unevenness of the grating preparation process.

One very interesting application of the PSTM is in measuring energy densities in optical waveguides. Fig. 5 shows a PSTM image of a "live" interference pattern seen on the surface of the prism by the PSTM. Light reflected from the exit face of the prism produces the interference with the incident beam. Such measurements together with profiles of the energy density in optical waveguides provide a novel application of the PSTM. The shape of interference fringes being wellknown, it is possible to use their measured shapes to determine the error introduced by the geometry of the probe tip.

Using photolithography we have made a number of crossed grating microstructure arrays. Fig. 6 shows a PSTM image of a crossed grating in photoresist while Fig. 7 shows the same microsturctures imaged by the PSTM after coating with silver. By playing around a bit with the interfering beams it is possible to make prolate spheroidal particles in an array. Fig. 8a shows an SEM image of such an array in silver-coated photoresist. While Fig. $8 \mathrm{~b}$ shows an ESTM image. Using this array as an etch mask we made a permanent sample etched into glass for repeated use in experiments on surface-enhanced Raman scattering. A PSTM image of this sample is shown in Fig. 8c. Other samples of use in Raman spectroscopy include polystyrene spheres. Fig. 9 shows a PSTM image of these. Spheres present special problems and benefits in PSTM work.

We have begun attempts to image biological samples, and while we presently are limited in the availability of such samples, a crude PSTM image of an E. coli bacterium is shown in Fig. 10. Thinner samples would be much easier to image and we soon hope to obtain a viral gel.

Finally, just for fun. an image of our quartz data was processed with Jifferent colors for different heights to make Fig. 11, which we call "Quartz Valley National Park".

5. PSTS (Photon Scanning-Tunneling Spectroscopy)

While the PSTM cannot touch the spatial resolution of the ESTM. it is well-known that optical spectroscopy has about the equivalent advantage over electron spectroscopy. We are able to carry out a variety of spectroscopies on PSTM samples using a split optical fïber. Absorption. Raman. and other spectroscopies can be carried out to permit chemical mapping and we expect this to be 
a major advantage for many samples. We recently made some measurements of localized fluorescence shifts on some samples brought to us by some colleagues and have begun examining variations in surface-enhanced Raman scattering on silver microstructures. Results are to be given elsewhere, but the work done thus far is very encouraging.

We have for some time been working with various microstructured silver substrates for use in surface-enhanced Raman spectroscopy (SERS). ${ }^{4}$ Our intent is now to detect the SERS signal of benzoic acid on the microstructures using a split fiber arrangement with the PSTM. It may be possible to detect the signal variation as a single one of the microstructures is scanned. 


\section{References}

1. R. C. Reddick, R. J. Warmack. T. L. Ferrell, Phys. Rev. B 39, 767 (1989).

2. For a complete discussion and additional references, see: R. C. Reddick, R. J. Warmack, D. W. Chilcott, S. L. Sharp, and T. L. Ferrell, Rev. Sci. Instrum. (in publication).

3. D. W. Pohl, W. Denk, and M. Lanz, Appl. Phys. Lett. 44, 651 (1984).

4. J. P. Goudonnet, T. Inagaki, T. L. Ferrell, R. J. Warmack, M. C. Buncick, and E. T. Arakawa, Chem. Phys. 106, 225 (1986). 


\section{FIGURE CAPTIONS}

Fig. 1 Schematic of PSTM

Fig. 2 PSTM image of a polished quartz surface.

Fig. 3 Images of a diffraction grating with $1.17 \mu \mathrm{m}$ line spacing: (a) PSTM image of grating in uncoated photoresist; (b) STM image of silver coated grating; and (c) SEM of silver coated grating.

Fig. 4 Cross-cut of the grating in Fig. 3.

Fig. 5 PSTM image of a "liven interference pattern

Fig. 6 PSTM image of a cross-grating in photoresist. The line spacing is one $1 \mu \mathrm{m}$.

Fig. 7 PSTM image of the silver-coated, crossed-grating of Fig. 6.

Fig. 8a SEM image of a "slewed-angle", crossed-grating in silver-coated photoresist. The line spacing is $1.7 \mu \mathrm{m}$.

Fig. 8b Electron STM image of the grating of the grating of Fig. 8a.

Fig. 8c The etched pattern left in glass by the grating of Fig. 8a as imaged by the PSTM.

Fig. 9 PSTM image of an array of $150 \mathrm{~nm}$ radius polystyrene spheres.

Fig. 10 PSTM image of E.coli.

Fig. 11 Quartz Valley National Park 


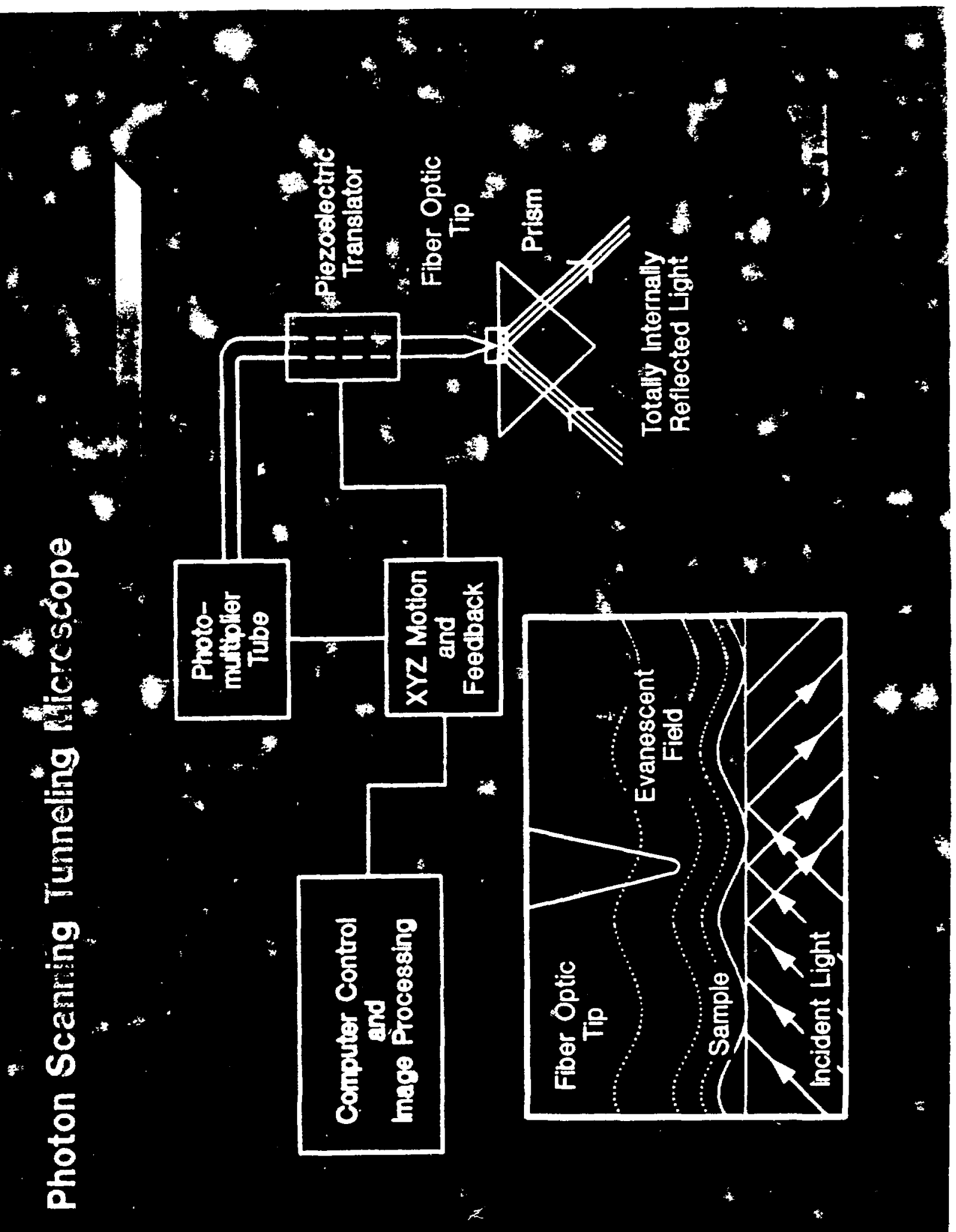




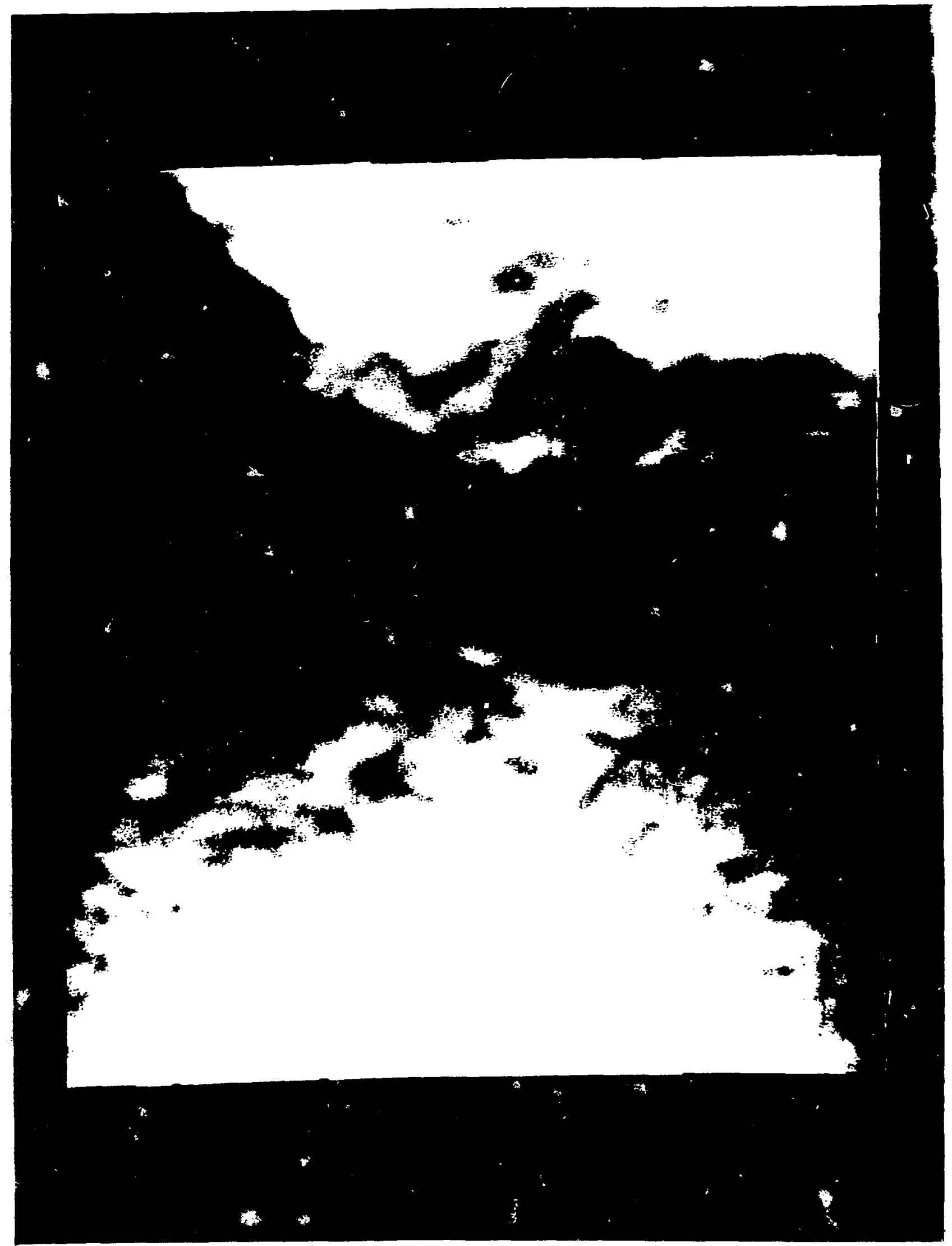




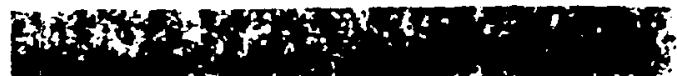
(n)

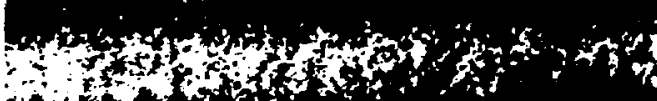
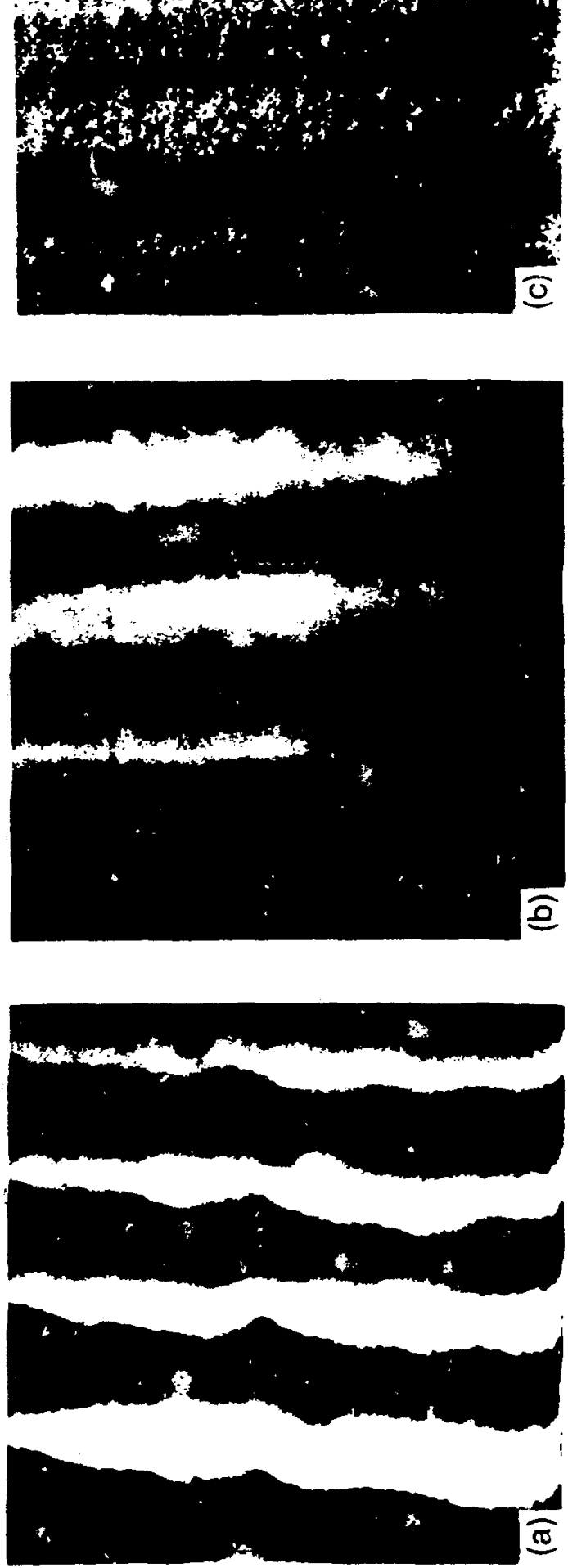


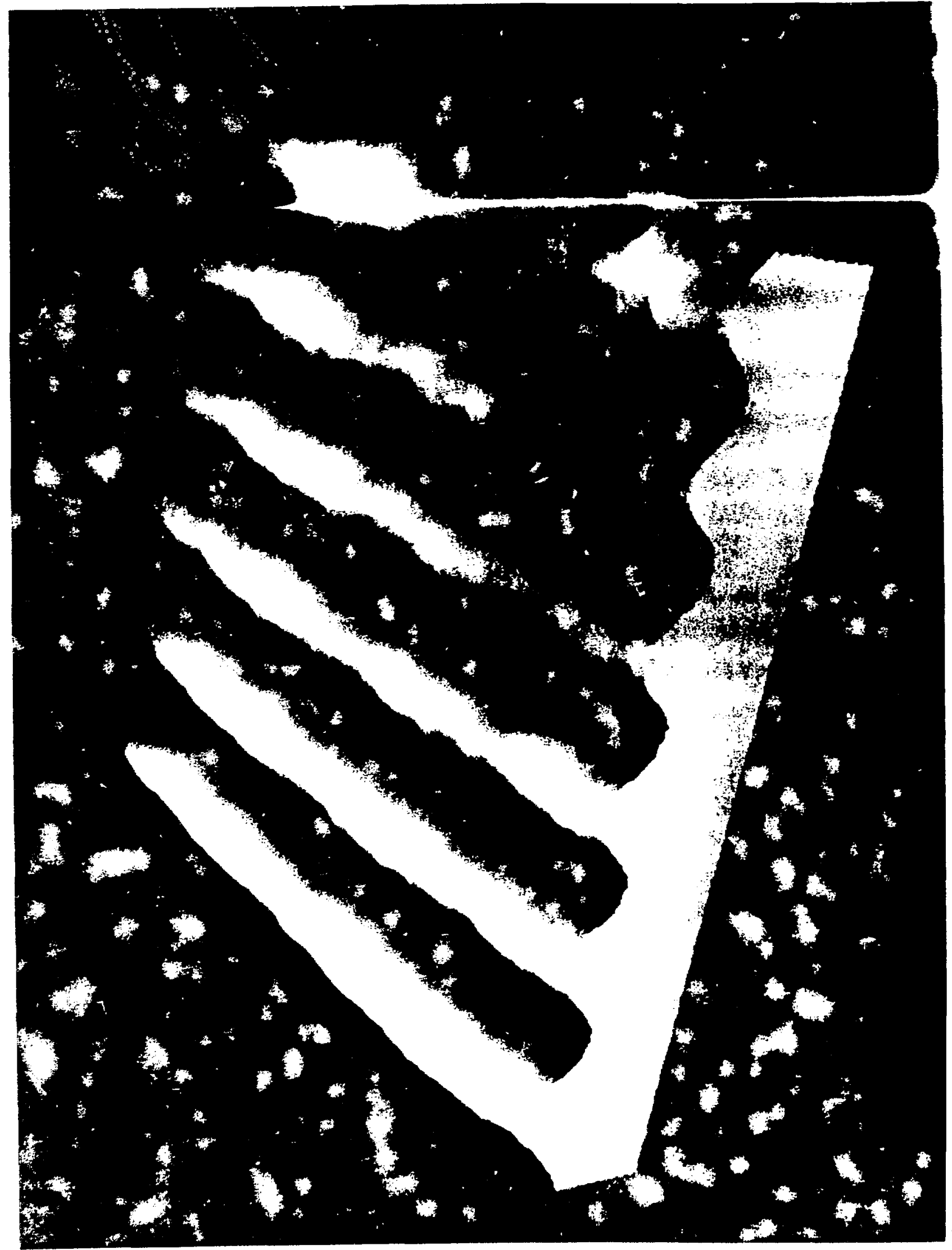




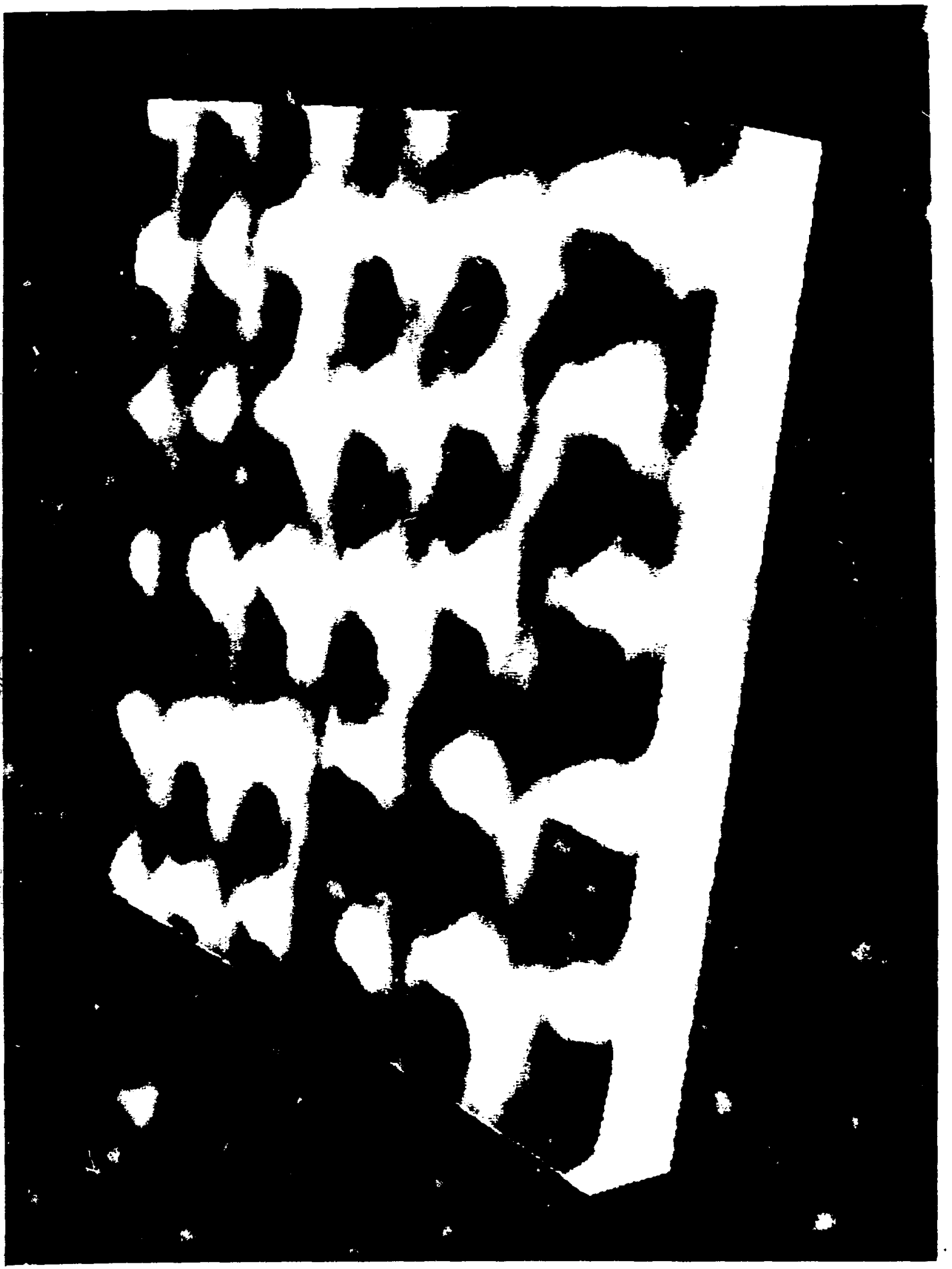




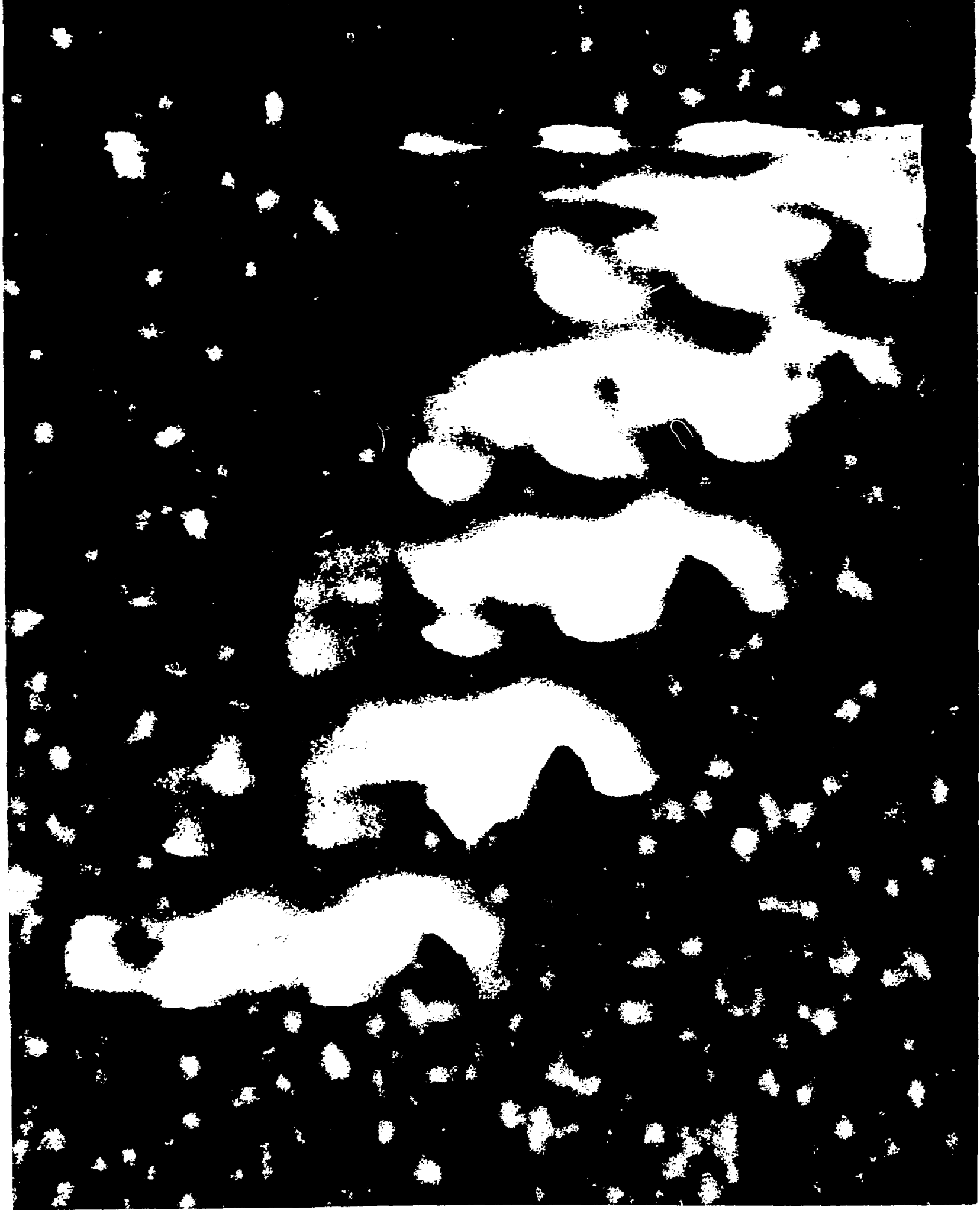




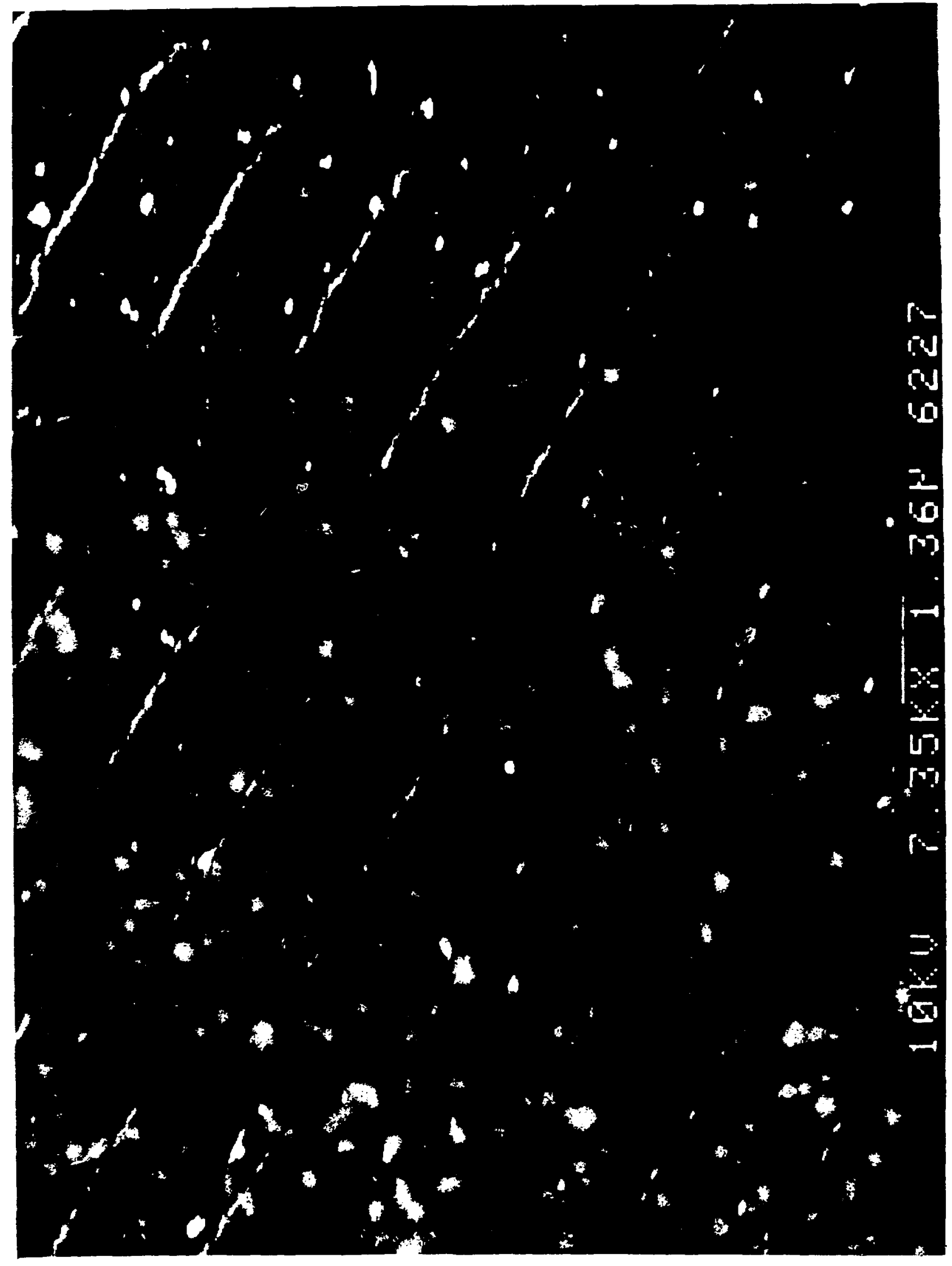


\&

:

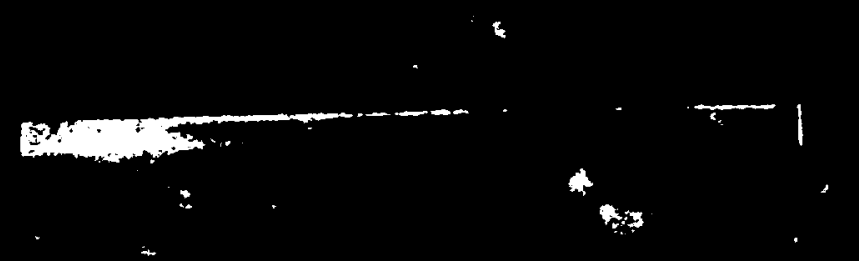

*.

y

$t^{6}$

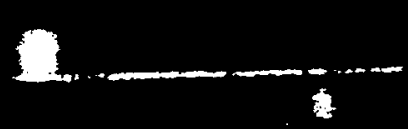

-

1

$i_{i \infty}^{\infty}+\infty$

3
1

$1+\ldots$

ta

$+\%$

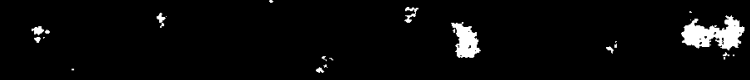

tin

$+\quad 4$

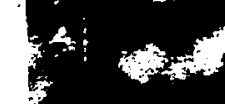

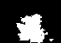

$x_{4}^{*}+\infty$

$1 . .4$

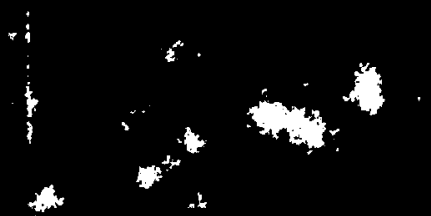

1

$y$

4 in

to

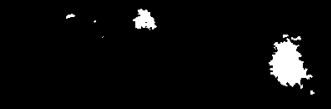

4

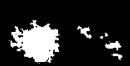

3

* : 


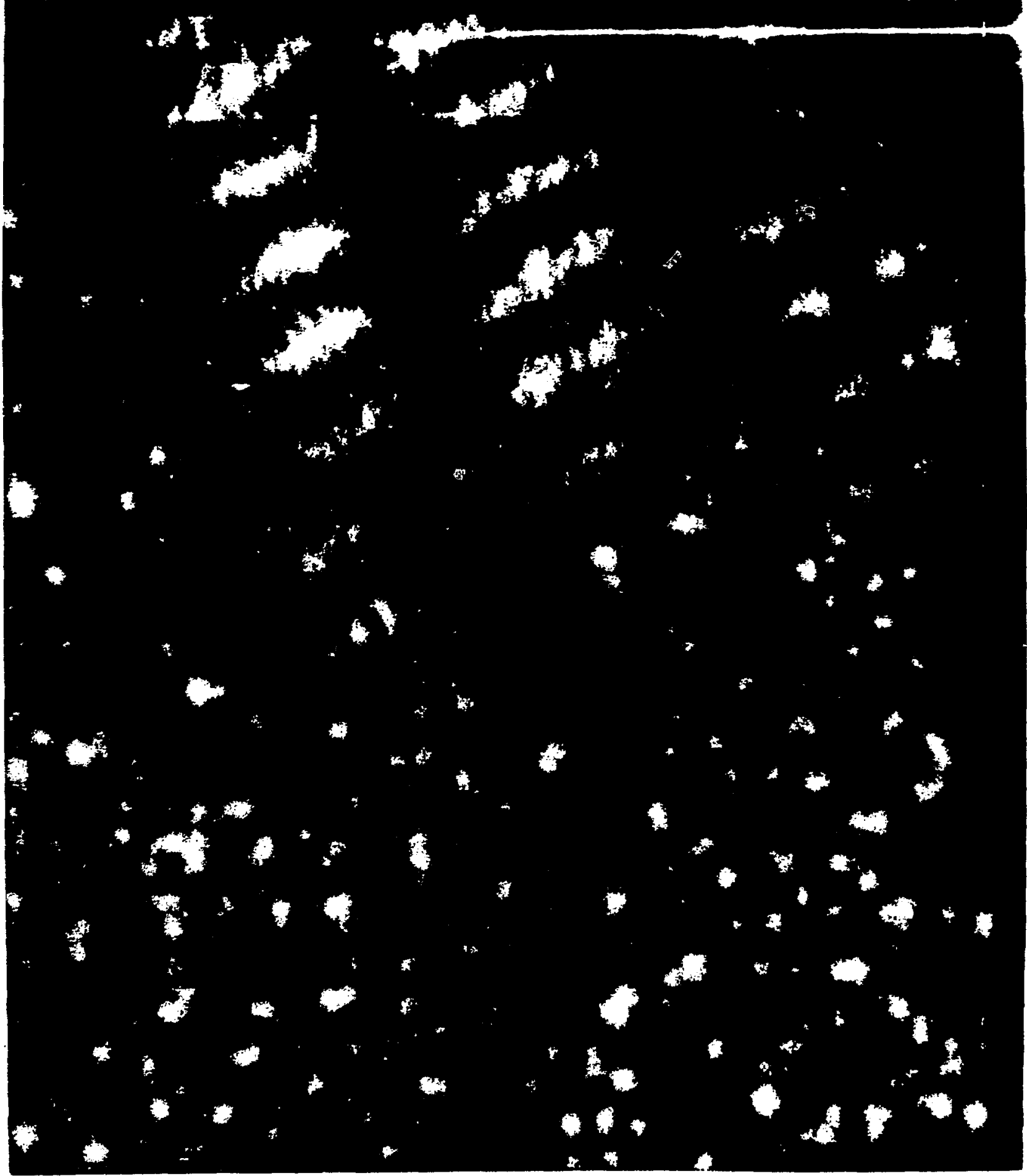


r
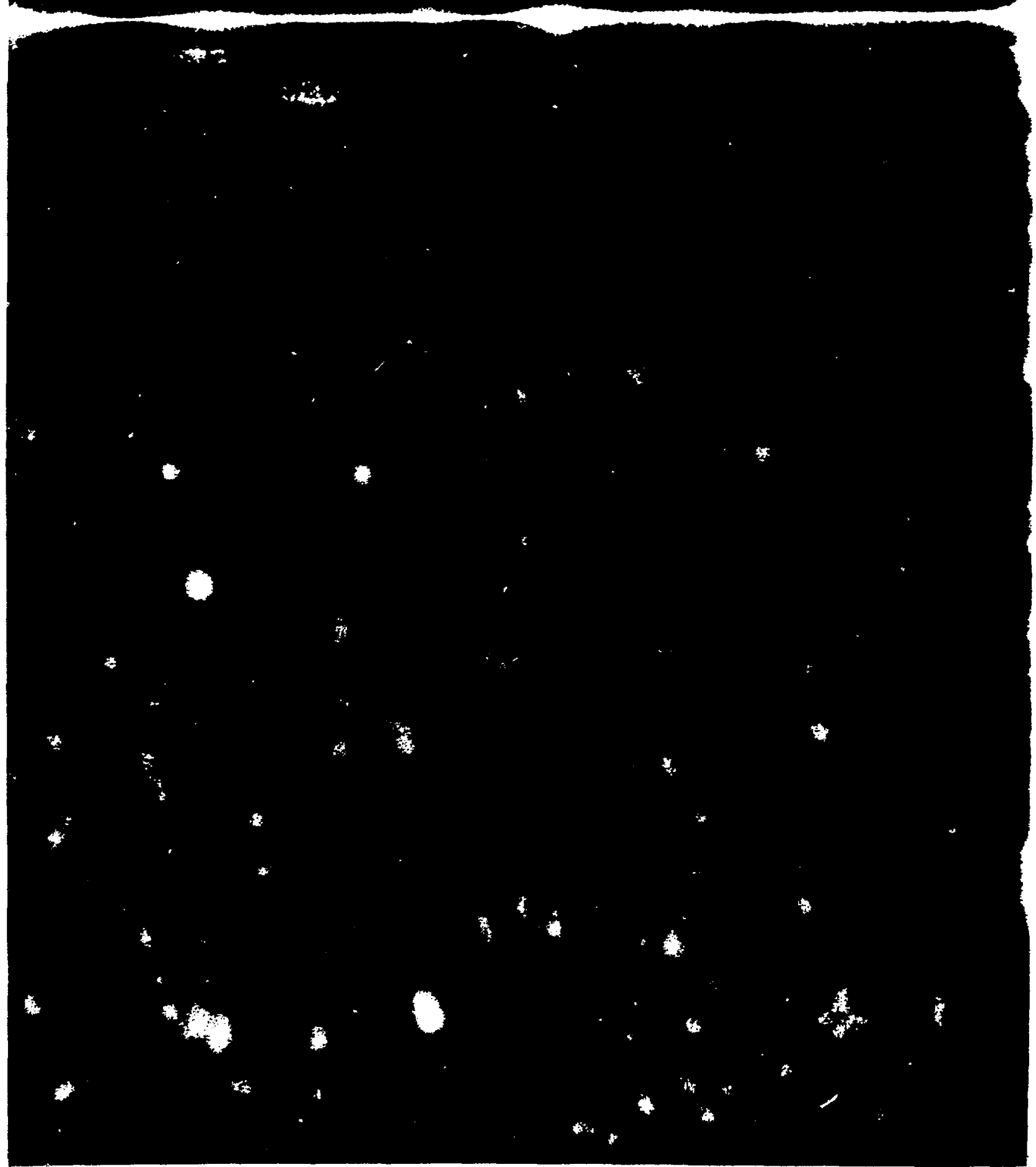


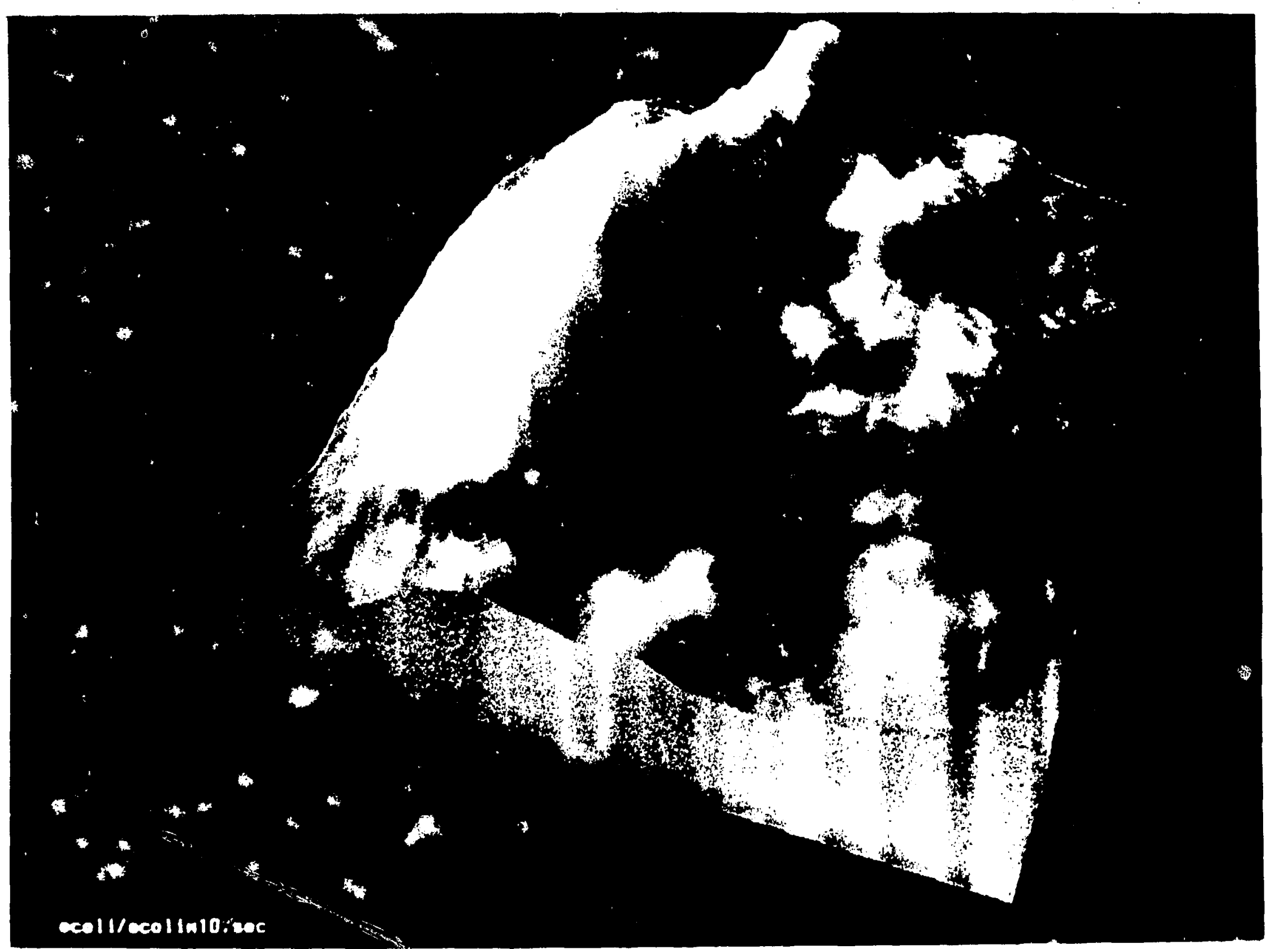




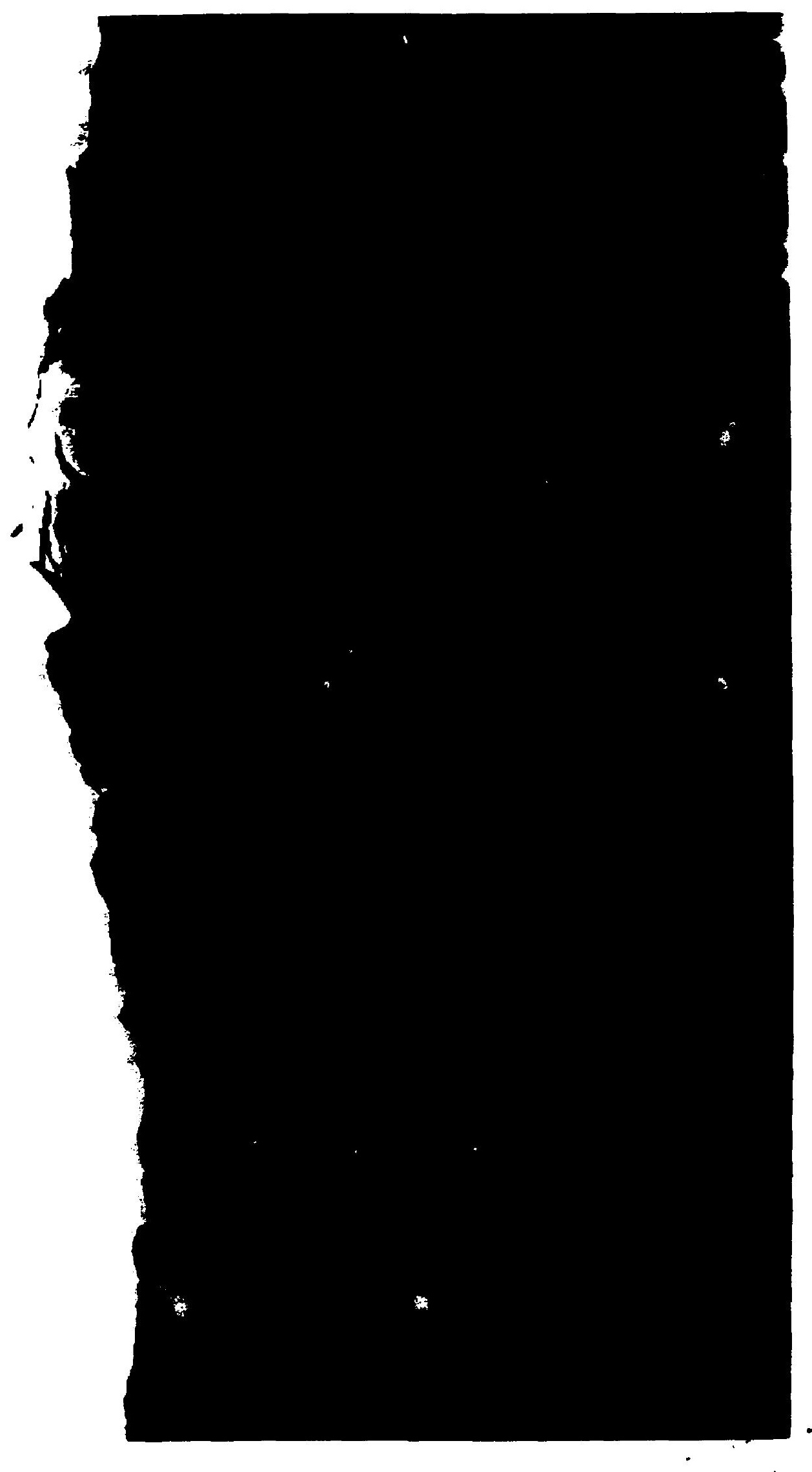

